\title{
$\mathrm{CuO} / \mathrm{CeO}_{2}-\mathrm{ZrO}_{2} / \mathrm{SiC}$ 整体催化剂催化甲醇水蒸气重整制氢的研究
}

\author{
焦桐 $a$ 许雪莲 ${ }^{a}$ 张䂞 $* a$ 翁幼云 $b$ 翁玉冰 $b$ 高志贤 $a$ \\ ( $a$ 辽宁石油化工大学 石油化工学院 抚顺 113001) \\ ( $b$ 贵州木易精细陶瓷有限公司 贵阳 550000)
}

\begin{abstract}
摘要 采用溶胶凝胶法制备了 $\mathrm{CeO}_{2}-\mathrm{ZrO}_{2}$ 固溶体载体涂层, 再经浸渍法制备了高空速下性能较好的甲醇水蒸气重整制 氢 $x \mathrm{CuO} / \mathrm{CeO}_{2}-\mathrm{ZrO}_{2} / \mathrm{SiC}$ 整体催化剂. 采用 $\mathrm{X}$ 射线衍射 $(\mathrm{XRD}) 、$ 比表面积测试 $(\mathrm{BET}) 、 \mathrm{H}_{2}$ 程序升温还原 $\left(\mathrm{H}_{2}-\mathrm{TPR}\right)$ 和 $\mathrm{X}$ 射 线光电子能谱(XPS)等手段对催化剂进行了表征, 结果表明, 催化剂活性主要与 $\mathrm{Cu}$ 比表面积、活性组分与载体之间相 互作用以及氧空穴量有关. 其中 $5 \% \mathrm{CuO} / \mathrm{CeO}_{2}-\mathrm{ZrO}_{2} / \mathrm{SiC}$ 整体催化剂的 $\mathrm{Cu}$ 比表面积较大, 活性组分 $\mathrm{CuO}$ 与 $\mathrm{CeO}_{2}-\mathrm{ZrO}_{2}$ 固溶体氧化物之间相互作用较强, 氧空穴量较多, 因此表现出较好的催化性能. 在反应温度为 $360{ }^{\circ} \mathrm{C}$ 、水醇物质的量 比为 1.2 、甲醇水蒸气气体空速为 $4840 \mathrm{~h}^{-1}$ 的条件下, 甲醇转化率为 $89.9 \%$, 产氢速率为 $1556 \mathrm{~L}^{\circ} \mathrm{m}^{-3} \cdot \mathrm{s}^{-1}$. 与传统颗粒催 化剂相比, $\mathrm{SiC}$ 基整体催化剂的产氢速率更高，更适用于高空速下的甲醇水蒸气重整制氢反应，有利于小型化制氢反应 器的集成.
\end{abstract}

关键词甲醇水蒸气重整; 氢气；一氧化碳; 固溶体; 整体催化剂

\section{Research on $\mathrm{CuO} / \mathrm{CeO}_{2}-\mathrm{ZrO}_{2} / \mathrm{SiC}$ Monolithic Catalysts for Hydrogen Production by Methanol Steam Reforming}

\author{
Tong Jiao $^{a} \quad$ Xue-lian Xu $^{a} \quad$ Lei Zhang*a $^{*, a} \quad$ You-yun Weng $^{b} \quad$ Yu-bing Weng $^{b} \quad$ Zhi-xian Gao $^{a}$ \\ ( ${ }^{a}$ School of Petrochemical Engineering, Liaoning Petrochemical University, Fushun 113001, China) \\ ( ${ }^{b}$ Guizhou Muyee Fine Ceramics Company Limited, Guiyang 550000, China)
}

\begin{abstract}
Hydrogen proton exchange membrane fuel cell is considered as the most cleanest way with high energy efficiency of hydrogen energy utilization. However, the high storage and transportation cost of hydrogen can always be the important factors hindering its development. Fortunately, on-site hydrogen production from methanol steam reforming can effectively solve this problem, which has low reforming temperature and CO content in reforming gas. Monolithic catalyst, a kind of catalytic material, on which the active components loaded on the formed support are generally honeycombed structures with multiple parallel channels. Therefore, compared to the traditional beaded catalytic materials, the monolithic catalytic material has lower pressure drop and higher mass transfer efficiency, which means that the monolithic catalysts have a certain practical application prospect. Ceria-zirconia solid solution was prepared by the sol-gel method, then a series $x \mathrm{CuO} / \mathrm{CeO}_{2}-\mathrm{ZrO}_{2} / \mathrm{SiC}$ catalysts were prepared by incipient-wetness impregnation method, and demonstrated considerable catalytic activity at high velocity reaction conditions. The catalyst samples were characterized by X-ray diffraction (XRD), BET specific surface area test, $\mathrm{H}_{2}$-temperature programmed reduction $\left(\mathrm{H}_{2}\right.$-TPR), X-ray photoelectron spectroscopy (XPS) techniques. According to the results, we proposed that the catalytic performances are mainly effected by the surface area of $\mathrm{Cu}$, the interaction between active component and support, and the amount of oxygen vacancy. Among those catalysts, $5 \% \mathrm{CuO} / \mathrm{CeO}_{2}-\mathrm{ZrO}_{2} / \mathrm{SiC}$ had relatively higher copper specific surface area, stronger interaction between $\mathrm{CuO}$ and support, more oxygen vacancies, thus showing better catalytic activity. When the reaction temperature reached $360{ }^{\circ} \mathrm{C}$, with a water/methanol molar ratio of 1.2 , methanol and water total gas hourly space velocity of $4840 \mathrm{~h}^{-1}$, the methanol conversion reached $89.9 \%$, and the $\mathrm{H}_{2}$ production rate was $1556 \mathrm{~L} \cdot \mathrm{m}^{-3} \cdot \mathrm{s}^{-1}$. When compared with the traditional bead catalyst, monolithic catalyst seems to be more suitable for high space velocity methanol steam reforming reaction, also the $\mathrm{SiC}$ support is chemical stable and has favorable thermal conductivity, which avoiding the device volume increase caused by the additional heating device, thus facilitating the integration of minitype reactors for hydrogen generation.

Keywords methanol steam reforming; hydrogen; carbon monoxide; solid solution; monolithic catalyst
\end{abstract}

\section{1 引言}

化石能源的使用和发展推动了人类文明的进步, 但
其燃烧时会释放大量有害物质，对自然环境造成了严重 的危害 ${ }^{[1-2]}$. 氢气作为新能源因其燃烧热值高, 燃烧产物 清洁等优点备受关注 ${ }^{[3]}$. 甲醇作为煤炭产业的副产品之

\footnotetext{
* E-mail: lnpuzhanglei@163.com

Received December 10, 2020; published February 1, 2021.

Project supported by the National Natural Science Foundation of China (No. 21673270).

项目受国家自然科学基金(No. 21673270)资助.
} 
一, 价格低廉且易于储存, 是良好的制氢原料. 甲醇制 氢方法一般分为三种, 即甲醇水蒸气重整制氢 ${ }^{[4]}$, 甲醇 部分氧化制氢 ${ }^{[5]}$ 和甲醇自热重整制氢 ${ }^{[6]}$. 其中甲醇水蒸 气重整制氢因其重整温度低且产物中 $\mathrm{CO}$ 含量较少等优 点而受到了广泛的关注 ${ }^{[7-9]}$. 目前, 用于甲醇水蒸气重整 制氢的催化剂主要是铜基颗粒催化材料 [10-13], 其中以 $\mathrm{CuO} / \mathrm{ZnO} / \mathrm{Al}_{2} \mathrm{O}_{3}$ 和 $\mathrm{CuO} / \mathrm{CeO}_{2}$ 颗粒催化材料 [14-15]最具代 表性. 颗粒催化材料存在压力降较大, 传热差, 温度场 分布不均匀等缺陷 ${ }^{[16]}$. 尤其对于强吸热的甲醇水蒸气 重整制氢反应，颗粒催化剂传热效率较低，造成温度场 分布不均匀, 且传质效率较低, 使得催化剂在高空速下 活性较差. 相较于传统的颗粒催化剂, 整体催化剂压力 降较小、温度分布相对均匀, 因此具备更好的应用性前 景 ${ }^{[17-19]}$. 刘娜等 ${ }^{[20-21]}$ 以堇青石为整体催化剂载体, $\mathrm{Ce}-\mathrm{Zr}$ 固溶体氧化物为涂层、 $\mathrm{ZnO}-\mathrm{Cr}_{2} \mathrm{O}_{3}$ 为活性组分制备了甲 醇自热重整制氢堇青石型整体催化剂, 探究了 $\mathrm{Zr}$ 的掺 杂对催化剂的影响. 结果表明, 相较于单一 $\mathrm{CeO}_{2}$ 涂层, 以 $\mathrm{Ce}-\mathrm{Zr}$ 固溶体为涂层的堇青石整体催化剂稳定运行时 间从 $67 \mathrm{~h}$ 提升至 $120 \mathrm{~h}$ 以上，甲醇转化率维持在 $100 \%$, 没有明显失活现象, 证明 $\mathrm{Ce}-\mathrm{Zr}$ 固溶体在兼具 $\mathrm{CeO}_{2}$ 优点 的同时, 还赋予催化剂更好的热稳定性, 可能是甲醇水 蒸气重整制氢催化剂的良好涂层之一. 然而堇青石陶瓷 导电性较差, 在集成为反应器时, 需要外加加热装置, 这就导致装置体积庞大且较为复杂, 仍有待改进. $\mathrm{SiC}$ 陶瓷载体在兼具传统陶瓷载体优点的基础上, 还具备良 好的导热性, 在集成为反应器时可实现通电自热, 这对 未来整体催化剂在移动氢源系统上的应用具有重要意 义, 因此以 $\mathrm{SiC}$ 作为整体催化剂的载体一方面有利于强 化传热, 另一方面有利于制氢反应器的集成, 可能是制 备甲醇水蒸气重整制氢整体催化剂的优秀载体材料之 一. 因此本工作通过溶胶凝胶法制备 $\mathrm{CeO}_{2}-\mathrm{ZrO}_{2}$ 固溶体 涂层，再利用浸渍法制备了 $\mathrm{CuO} / \mathrm{CeO}_{2}-\mathrm{ZrO}_{2} / \mathrm{SiC}$ 整体催 化剂，探讨 $\mathrm{Cu}$ 含量对甲醇水蒸气重整制氢 $\mathrm{CuO} /$ $\mathrm{CeO}_{2}-\mathrm{ZrO}_{2} / \mathrm{SiC}$ 整体催化剂结构及性能的影响, 为整体 催化剂在甲醇水蒸气重整制氢反应中的应用提供基础 数据.

\section{2 结果与讨论}

\section{$2.1 \mathrm{SiC}$ 陶瓷载体参数}

本工作所用的 $\mathrm{SiC}$ 陶瓷载体整体直径为 $8.2 \mathrm{~mm}$, 表 面均匀分布 200 个小孔, 每个孔直径约为 $0.50 \sim 0.56$ $\mathrm{mm}$, 几何面积为 $12.51 \mathrm{~cm}^{2}$, 常温导热系数为 490 $\mathrm{W} \cdot \mathrm{m}^{-1} \cdot \mathrm{K}^{-1}$. 表 1 为 $\mathrm{SiC}$ 与其他常用导热材料常温下导 热系数对比表, 说明 $\mathrm{SiC}$ 具有良好的导热性能.

\section{$2.2 \mathrm{XRD}$ 分析}

图 1 为 $\mathrm{CeO}_{2}-\mathrm{ZrO}_{2} / \mathrm{SiC}$ 整体催化材料和 $x \mathrm{CuO} /$ $\mathrm{CeO}_{2}-\mathrm{ZrO}_{2} / \mathrm{SiC}$ 整体催化剂的 XRD 谱图. 从图 1 中可知,
表 1 导热系数对比表

Table 1 Comparison table of thermal conductivity

\begin{tabular}{cc}
\hline 材料 & 导热系数 $/\left(\mathrm{W} \cdot \mathrm{m}^{-1} \cdot \mathrm{K}^{-1}\right)$ \\
\hline $\mathrm{SiC}$ & 490 \\
纯铜 & 401 \\
纯金 & 317 \\
纯铝 & 237 \\
\hline
\end{tabular}

所有催化材料并没有出现 $\mathrm{ZrO}_{2}$ 的特征衍射峰, 并且在 $28.6^{\circ} 、 33.1^{\circ} 、 47.5^{\circ}$ 和 $56.4^{\circ}$ 处的 $\mathrm{CeO}_{2}$ 特征衍射峰均向 高角度偏移, 这是由于 $\mathrm{Zr}^{4}$ 进入了 $\mathrm{CeO}_{2}$ 的晶格中, 使 $\mathrm{CeO}_{2}$ 的晶格形成了表面缺陷, 形成 $\mathrm{CeO}_{2}-\mathrm{ZrO}_{2}$ 固溶体造 成的 ${ }^{[22-23]}$. 另外通过与 $\mathrm{CuO}$ 的特征衍射峰对比, 发现 $2 \% \mathrm{CuO} / \mathrm{CeO}_{2}-\mathrm{ZrO}_{2} / \mathrm{SiC}$ 整体催化剂并没有出现 $\mathrm{CuO}$ 的 衍射峰, 这可能是由于 $\mathrm{CuO}$ 在 $\mathrm{CeO}_{2}-\mathrm{ZrO}_{2}$ 固溶体表面含 量较少, $\mathrm{CuO}$ 以高度分散的形式存在造成的 ${ }^{[24]}$. 当 $\mathrm{CuO}$ 负载量达到 $5 \%$ 后, 开始出现 $\mathrm{CuO}$ 的特征衍射峰, 并且 随着 $\mathrm{Cu}$ 含量的增加, 其特征衍射峰越来越明显.

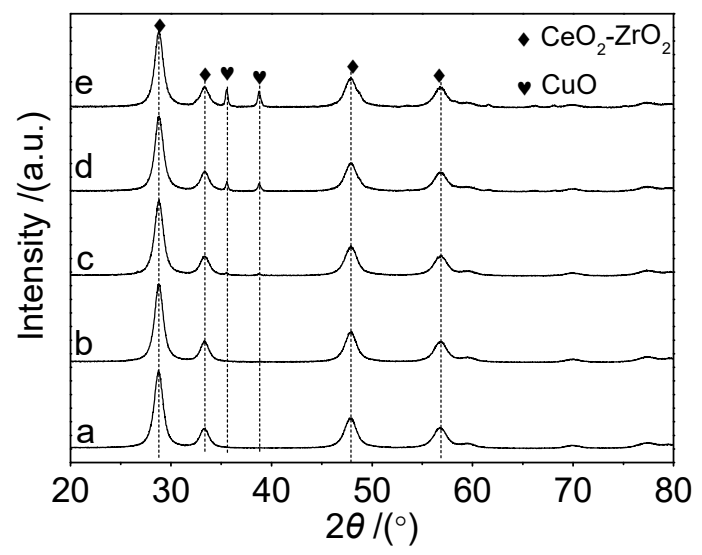

图 $1 x \mathrm{CuO} / \mathrm{CeO}_{2}-\mathrm{ZrO}_{2}$ 催化剂的 XRD 谱图

Figure $1 \mathrm{XRD}$ spectra of $x \mathrm{CuO} / \mathrm{CeO}_{2}-\mathrm{ZrO}_{2} / \mathrm{SiC}$ catalysts

(a) $\mathrm{CeO}_{2}-\mathrm{ZrO}_{2} ;$ (b) $2 \% \mathrm{CuO} / \mathrm{CeO}_{2}-\mathrm{ZrO}_{2} / \mathrm{SiC}$; (c) $5 \% \mathrm{CuO} / \mathrm{CeO}_{2}-\mathrm{ZrO}_{2} / \mathrm{SiC}$; (d) $10 \% \mathrm{CuO} / \mathrm{CeO}_{2}-\mathrm{ZrO}_{2} / \mathrm{SiC}$; (e) $20 \% \mathrm{CuO} / \mathrm{CeO}_{2}-\mathrm{ZrO}_{2} / \mathrm{SiC}$

\section{3 催化剂表面性质分析}

从表 2 中可以看到, 未负载 $\mathrm{CuO}$ 的 $\mathrm{CeO}_{2}-\mathrm{ZrO}_{2}$ 载体 比表面积为 $98.3 \mathrm{~m}^{2} \cdot \mathrm{g}^{-1}$; 负载少量 $\mathrm{CuO}$ 后, 所得 $2 \% \mathrm{CuO} / \mathrm{CeO}_{2}-\mathrm{ZrO}_{2} / \mathrm{SiC}$ 催化剂比表面积没有明显的变 化; 随着 $\mathrm{Cu}$ 含量增加, 催化剂的比表面积逐渐减小, 这 是由于 $\mathrm{Cu}$ 含量增大, $\mathrm{CuO}$ 占据了 $\mathrm{CeO}_{2}-\mathrm{ZrO}_{2}$ 固溶体中的 孔道造成的 ${ }^{[25]}$, 这与其孔容减小是一致的. 表 2 还列出 了 $\mathrm{Cu}$ 比表面, 可以看到 $5 \% \mathrm{CuO} / \mathrm{CeO}_{2}-\mathrm{ZrO}_{2} / \mathrm{SiC}$ 催化剂 的最大, 为 $5.8 \mathrm{~m}^{2} \cdot \mathrm{g}^{-1}$; 其次为 $10 \% \mathrm{CuO} / \mathrm{CeO}_{2}-\mathrm{ZrO}_{2} / \mathrm{SiC}$ 和 $20 \% \mathrm{CuO} / \mathrm{CeO}_{2}-\mathrm{ZrO}_{2} / \mathrm{SiC}$, 分别为 5.6 和 $5.1 \mathrm{~m}^{2} \cdot \mathrm{g}^{-1}$, 最 后 $2 \% \mathrm{CuO} / \mathrm{CeO}_{2}-\mathrm{ZrO}_{2} / \mathrm{SiC}$ 催化剂 $\mathrm{Cu}$ 比表面积最小, 为 $4.8 \mathrm{~m}^{2} \cdot \mathrm{g}^{-1}$; 这是因为当 $\mathrm{Cu}$ 含量较低 $(2 \% \mathrm{CuO} /$ $\mathrm{CeO}_{2}-\mathrm{ZrO}_{2} / \mathrm{SiC}$ 催化剂)时, 催化剂表面 $\mathrm{CuO}$ 含量较少, 不足以提供较大的 $\mathrm{Cu}$ 比表面积; 而当 $\mathrm{Cu}$ 负载量增加到 一定程度时 $\left(10 \% \mathrm{CuO} / \mathrm{CeO}_{2}-\mathrm{ZrO}_{2} / \mathrm{SiC}\right.$ 催化剂 $) \mathrm{Cu}$ 会聚集 
长大, 进而使得 $\mathrm{Cu}$ 比表面积降低. 另外, 从表 2 中还可 以看出, 催化剂 $\mathrm{Cu}$ 比表面积与产氢速率相对应, $5 \% \mathrm{CuO} / \mathrm{CeO}_{2}-\mathrm{ZrO}_{2} / \mathrm{SiC}$ 催化剂的 $\mathrm{Cu}$ 比表面积最大, 对 应的产氢速率最高, 为 $1556 \mathrm{~L} \cdot \mathrm{m}^{-3} \cdot \mathrm{s}^{-1}$, 这说明催化剂 $\mathrm{Cu}$ 的比表面积是影响催化剂活性的重要因素之一.

表 2 催化剂的物化性质和产氢速率

Table 2 Physicochemical and hydrogen production rate of catalysts

\begin{tabular}{|c|c|c|c|c|}
\hline 催化剂 ${ }^{a}$ & $\begin{array}{l}\text { 比表面积/ } \\
\left(\mathrm{m}^{2} \cdot \mathrm{g}^{-1}\right)\end{array}$ & $\begin{array}{c}\text { 孔容/ } \\
\left(\mathrm{cm}^{3} \cdot \mathrm{g}^{-1}\right)\end{array}$ & $\begin{array}{c}\mathrm{Cu} \text { 比表面积 } b / \\
\left(\mathrm{m}^{2} \bullet \mathrm{g}^{-1}\right)\end{array}$ & $\begin{array}{l}\text { 产氢速率 } c / \\
\left(\mathrm{L} \cdot \mathrm{m}^{-3} \cdot \mathrm{s}^{-1}\right)\end{array}$ \\
\hline $\mathrm{a}$ & 98.3 & 0.32 & - & - \\
\hline b & 98.3 & 0.31 & 4.8 & 631 \\
\hline $\mathrm{c}$ & 94.6 & 0.30 & 5.8 & 1556 \\
\hline d & 69.5 & 0.26 & 5.6 & 1418 \\
\hline $\mathrm{e}$ & 63.2 & 0.21 & 5.1 & 1407 \\
\hline
\end{tabular}

a (a) $\mathrm{CeO}_{2}-\mathrm{ZrO}_{2} ;$ (b) $2 \% \mathrm{CuO} / \mathrm{CeO}_{2}-\mathrm{ZrO}_{2} / \mathrm{SiC}$; (c) $5 \% \mathrm{CuO} / \mathrm{CeO}_{2}-\mathrm{ZrO}_{2} / \mathrm{SiC}$; (d) $10 \% \mathrm{CuO} / \mathrm{CeO}_{2}-\mathrm{ZrO}_{2} / \mathrm{SiC}$; (e) $20 \% \mathrm{CuO} / \mathrm{CeO}_{2}-\mathrm{ZrO}_{2} / \mathrm{SiC}$. ${ }^{b}$ Determined by $\mathrm{N}_{2} \mathrm{O}$ experiment. ${ }^{c}$ Reaction temperature: $360{ }^{\circ} \mathrm{C}$, water and methanol molar ratio: 1.2 , GHSV: $4840 \mathrm{~h}^{-1}$.

\section{$2.4 \mathrm{H}_{2}-\mathrm{TPR}$ 分析}

为了研究 $x \mathrm{CuO} / \mathrm{CeO}_{2}-\mathrm{ZrO}_{2} / \mathrm{SiC}$ 整体催化剂的还原 性能, 进行了 $\mathrm{H}_{2}-\mathrm{TPR}$ 分析, 图 2 为 $x \mathrm{CuO} / \mathrm{CeO}_{2}-\mathrm{ZrO}_{2} / \mathrm{SiC}$ 整体催化剂的 $\mathrm{H}_{2}$-TPR 谱图, 图中的所有还原峰均为不 同 $\mathrm{CuO}$ 物种的还原峰. 四种 $x \mathrm{CuO} / \mathrm{CeO}_{2}-\mathrm{ZrO}_{2} / \mathrm{SiC}$ 整体 催化剂均出现了两个还原峰, 低温位置的 $\alpha$ 峰是表相 $\mathrm{CuO}$ 的还原峰; 高温位置的 $\beta$ 峰是体相 $\mathrm{CuO}$ 的还原峰.

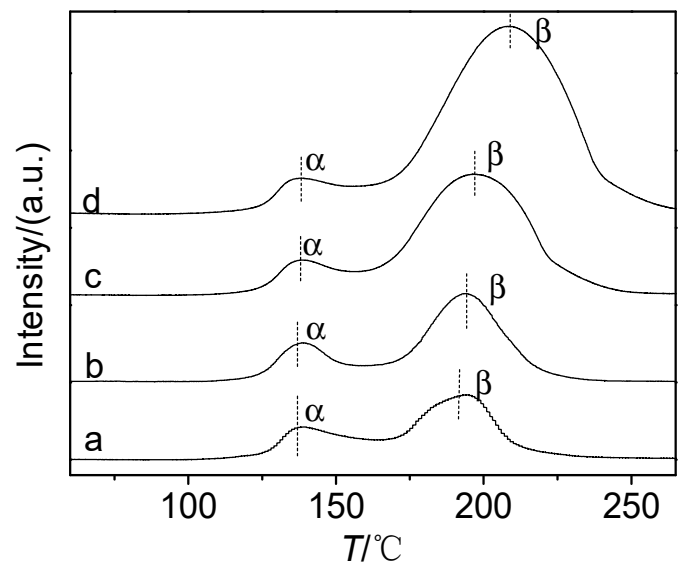

图 $2 x \mathrm{CuO} / \mathrm{CeO}_{2}-\mathrm{ZrO}_{2} / \mathrm{SiC}$ 整体催化剂的 $\mathrm{H}_{2}$-TPR 谱图 Figure $2 \mathrm{H}_{2}$-TPR spectra of $x \mathrm{CuO} / \mathrm{CeO}_{2}-\mathrm{ZrO}_{2} / \mathrm{SiC}$ catalysts (a) $2 \% \mathrm{CuO} / \mathrm{CeO}_{2}-\mathrm{ZrO}_{2} / \mathrm{SiC}$; (b) $5 \% \mathrm{CuO} / \mathrm{CeO}_{2}-\mathrm{ZrO}_{2} / \mathrm{SiC}$; (c) $10 \% \mathrm{CuO} /$ $\mathrm{CeO}_{2}-\mathrm{ZrO}_{2} / \mathrm{SiC}$; (d) $20 \% \mathrm{CuO} / \mathrm{CeO}_{2}-\mathrm{ZrO}_{2} / \mathrm{SiC}$

表 3 给出了各还原峰的温度和峰面积占比, 结果表 明, $2 \% \mathrm{CuO} / \mathrm{CeO}_{2}-\mathrm{ZrO}_{2} / \mathrm{SiC}$ 和 $5 \% \mathrm{CuO} / \mathrm{CeO}_{2}-\mathrm{ZrO}_{2} / \mathrm{SiC}$ 整 体催化剂还原峰温度较低, 这是因为催化剂中 $\mathrm{CuO}$ 与 $\mathrm{CeO}_{2}-\mathrm{ZrO}_{2}$ 固溶体相互作用较强, $\mathrm{CuO}$ 分散较好, 因此 更容易被还原. 根据本课题组前期研究成果表明, 活性 组分与载体之间相互作用越强, 催化活性越好 ${ }^{[26]}$. 但结 合图 8 发现， $2 \% \mathrm{CuO} / \mathrm{CeO}_{2}-\mathrm{ZrO}_{2} / \mathrm{SiC}$ 和 $5 \% \mathrm{CuO} /$ $\mathrm{CeO}_{2}-\mathrm{ZrO}_{2} / \mathrm{SiC}$ 整体催化剂的活性相差很大, 这是因为
$2 \% \mathrm{CuO} / \mathrm{CeO}_{2}-\mathrm{ZrO}_{2} / \mathrm{SiC}$ 催化剂中 $\mathrm{CuO}$ 负载量较小，导致 催化剂的 $\mathrm{Cu}$ 比表面积较小, 起到催化活性作用的活性 位较少, 进而催化活性较差. 而 $5 \% \mathrm{CuO} / \mathrm{CeO}_{2}-\mathrm{ZrO}_{2} / \mathrm{SiC}$ 整体催化剂中 $\mathrm{Cu}$ 比表面积较大, $\mathrm{CuO}$ 与 $\mathrm{CeO}_{2}-\mathrm{ZrO}_{2}$ 固溶 体相互作用较强, 表相 $\mathrm{CuO}$ 占比较大, 因此催化活性较 好. 除此之外, 随着 $\mathrm{CuO}$ 负载量的增加, $\beta$ 峰还原温度逐 渐上升. 这是因为随着 $\mathrm{CuO}$ 负载量的增加, $\mathrm{CuO}$ 的分散 变差, $\mathrm{CuO}$ 与 $\mathrm{CeO}_{2}-\mathrm{ZrO}_{2}$ 固溶体载体之间相互作用减弱 所导致的. 由此可见, 载体与活性组分相互作用越强, 表相 $\mathrm{CuO}$ 越多, $\mathrm{Cu}$ 的比表面积越大, 催化活性越好.

表 $3 \mathrm{CuO}$ 还原峰位置及峰面积占比

Table 3 Reduction peak positions and area content of $\mathrm{CuO}$

\begin{tabular}{ccccc}
\hline \multirow{2}{*}{ 催化剂 } & \multicolumn{2}{c}{ 峰位置 $/{ }^{\circ} \mathrm{C}$} & \multicolumn{2}{c}{ 面积占比 $/ \%$} \\
\cline { 2 - 5 } & $\alpha$ 峰 & $\beta$ 峰 & $\alpha$ 峰 & $\beta$ 峰 \\
\hline $2 \% \mathrm{CuO} / \mathrm{CeO}_{2}-\mathrm{ZrO}_{2} / \mathrm{SiC}$ & 137 & 192 & $30.2 \%$ & $69.8 \%$ \\
$5 \% \mathrm{CuO} / \mathrm{CeO}_{2}-\mathrm{ZrO}_{2} / \mathrm{SiC}$ & 137 & 194 & $24.6 \%$ & $75.4 \%$ \\
$10 \% \mathrm{CuO} / \mathrm{CeO}_{2}-\mathrm{ZrO}_{2} / \mathrm{SiC}$ & 138 & 197 & $14.3 \%$ & $85.7 \%$ \\
$20 \% \mathrm{CuO} / \mathrm{CeO}_{2}-\mathrm{ZrO}_{2} / \mathrm{SiC}$ & 138 & 208 & $9.0 \%$ & $91.0 \%$ \\
\hline
\end{tabular}

\subsection{XPS 分析}

为测定催化剂表面物种原子价态，本工作引入 $X$ 射 线光电子能谱分析(XPS), 以此来探究原子价态对催化 剂性能的影响.

\subsubsection{Ce 3d XPS 分析}

图 3 为 $x \mathrm{CuO} / \mathrm{CeO}_{2}-\mathrm{ZrO}_{2} / \mathrm{SiC}$ 整体催化剂的 $\mathrm{Ce} 3 \mathrm{~d} \mathrm{X}$ 射线光电子能谱. 图中每种催化剂均出现了 8 个 $\mathrm{Ce} 3 \mathrm{~d}$ 自旋轨道的特征峰，其中, $u 、 u^{\prime} 、 u^{\prime \prime} 、 u^{\prime \prime}$ 归属于 Ce $3 d_{3 / 2}$ 能级的电子结合能，而 $v 、 v^{\prime} 、 v^{\prime \prime} 、 v^{\prime \prime}$ 则归属于 $\mathrm{Ce} 3 \mathrm{~d}_{5 / 2}$ 能级的电子结合能. 另外在这 8 个峰中, $\mathrm{u}^{\prime}$ 和 $\mathrm{v}^{\prime}$ 归属于 $\mathrm{Ce}^{3^{+}}$, 其余均归属于 $\mathrm{Ce}^{4^{+}[27]} . \mathrm{Ce}^{3^{+}}$的产生是由于 $\mathrm{CeO}_{2}-\mathrm{ZrO}_{2}$ 固溶体中的 $\mathrm{Ce}^{4+}$ 与 $\mathrm{Cu}^{+}$之间发生了相互作用, 致使 $\mathrm{Ce}^{4+}$ 被还原为 $\mathrm{Ce}^{3+}$ 所致 ${ }^{[28]}$. 除此之外, 根据表面电

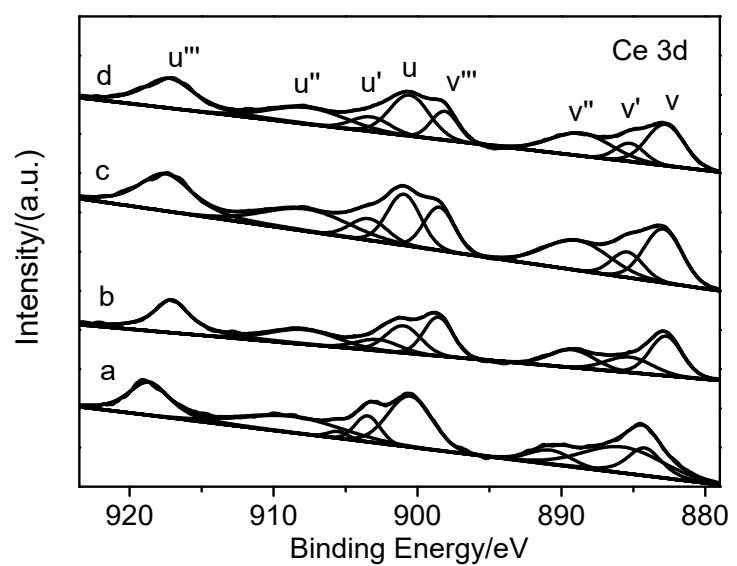

图 $3 x \mathrm{CuO} / \mathrm{CeO}_{2}-\mathrm{ZrO}_{2} / \mathrm{SiC}$ 整体催化剂 $\mathrm{Ce} 3 \mathrm{~d}$ XPS 谱图

Figure $3 \mathrm{Ce} 3 \mathrm{~d}$ XPS spectra of $x \mathrm{CuO} / \mathrm{CeO}_{2}-\mathrm{ZrO}_{2} / \mathrm{SiC}$ catalysts (a) $2 \% \mathrm{CuO} / \mathrm{CeO}_{2}-\mathrm{ZrO}_{2} / \mathrm{SiC}$; (b) $5 \% \mathrm{CuO} / \mathrm{CeO}_{2}-\mathrm{ZrO}_{2} / \mathrm{SiC}$; (c) $10 \% \mathrm{CuO} /$ $\mathrm{CeO}_{2}-\mathrm{ZrO}_{2} / \mathrm{SiC}$; (d) $20 \% \mathrm{CuO} / \mathrm{CeO}_{2}-\mathrm{ZrO}_{2} / \mathrm{SiC}$ 
荷守恒原理, $\mathrm{Ce}^{3+}$ 的出现也表明催化剂表面存在氧空穴, 而氧空穴的生成对氧化还原反应是有利的 ${ }^{[29]}$.

表 4 为四种催化剂的 $\mathrm{Ce}$ 和 $\mathrm{Cu}$ 的 XPS 曲线拟合结 果. 表中数据表明, $5 \% \mathrm{CuO} / \mathrm{CeO}_{2}-\mathrm{ZrO}_{2} / \mathrm{SiC}$ 整体催化剂 中 $\mathrm{Ce}^{3+}$ 含量较高, 说明其中 $\mathrm{Ce}-\mathrm{Cu}$ 之间相互作用较强、 $\mathrm{CeO}_{2}-\mathrm{ZrO}_{2}$ 固溶体载体中氧空穴较多. 并且, 四种催化 剂 $\mathrm{Ce}^{3+}$ 含量占比大小顺序与催化活性强弱顺序一致, 由此可见, 催化剂中 $\mathrm{Ce}-\mathrm{Cu}$ 相互作用强度和氧空穴数量 是决定催化剂催化活性的重要因素.

表 4 催化剂 $\mathrm{Ce}$ 和 $\mathrm{Cu}$ 的 XPS 曲线拟合结果

Table 4 Fitting results of $\mathrm{Cu}$ and Ce XPS curves of catalysts

\begin{tabular}{ccc}
\hline 催化剂 & $\mathrm{Ce}^{3+}$ 含量占比 $/ \%$ & $\mathrm{Cu}^{+}$含量占比 $/ \%$ \\
\hline $2 \% \mathrm{CuO} / \mathrm{CeO}_{2}-\mathrm{ZrO}_{2} / \mathrm{SiC}$ & 9.9 & 67.1 \\
$5 \% \mathrm{CuO} / \mathrm{CeO}_{2}-\mathrm{ZrO}_{2} / \mathrm{SiC}$ & 14.5 & 70.5 \\
$10 \% \mathrm{CuO} / \mathrm{CeO}_{2}-\mathrm{ZrO}_{2} / \mathrm{SiC}$ & 12.3 & 67.3 \\
$20 \% \mathrm{CuO} / \mathrm{CeO}_{2}-\mathrm{ZrO}_{2} / \mathrm{SiC}$ & 11.2 & 59.2 \\
\hline
\end{tabular}

\subsubsection{Cu 2p 和 Cu LMM XPS 分析}

图 4 为 $x \mathrm{CuO} / \mathrm{CeO}_{2}-\mathrm{ZrO}_{2} / \mathrm{SiC}$ 整体催化剂的 $\mathrm{Cu} 2 \mathrm{p} \mathrm{X}$ 射线光电子能谱. 图中 $944 \mathrm{eV}$ 附近的特征峰为 $\mathrm{Cu} 2 \mathrm{p}$ 的 卫星峰, 该峰归属于 $\mathrm{Cu}^{2+}$, 说明催化剂表面存在 $\mathrm{CuO}$ 物 种, 与 XRD 表征结果相符. 而 $934 \mathrm{eV}$ 附近的特征峰和 $954 \mathrm{eV}$ 附近的特征峰则分别对应 $\mathrm{Cu} 2 \mathrm{p}_{3 / 2}$ 能级和 $\mathrm{Cu}$ $2 p_{1 / 2}$ 能级的电子结合能, 并且以上两种特征峰均归属于 $\mathrm{Cu}^{+}$或 $\mathrm{Cu}^{0}$. 但 $\mathrm{Cu}^{+}$和 $\mathrm{Cu}^{0}$ 两物种的出峰位置与特征峰形 状均较为类似, 为两种物质的辨别造成了困难, 因此加 入了 $\mathrm{Cu} \mathrm{LMM}$ 谱图, 以进一步区分 $\mathrm{Cu}^{+}$和 $\mathrm{Cu}^{0}$ 物种.

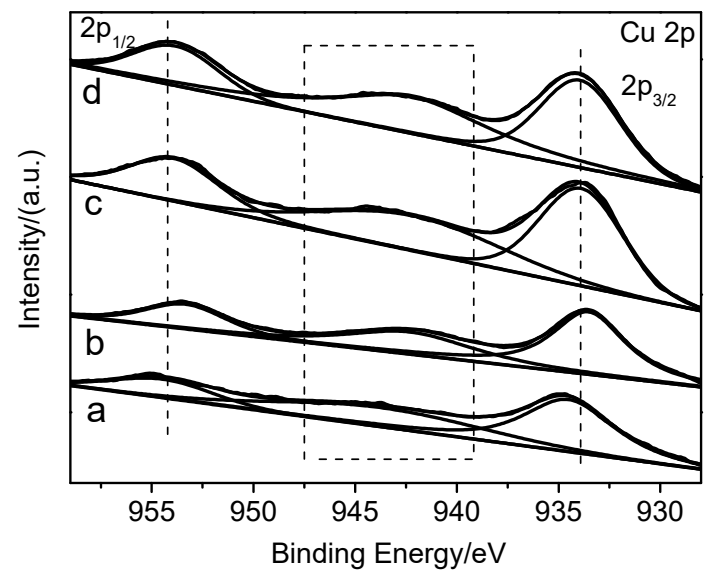

图 $4 x \mathrm{CuO} / \mathrm{CeO}_{2}-\mathrm{ZrO}_{2} / \mathrm{SiC}$ 整体催化剂 $\mathrm{Cu} 2 \mathrm{p} \mathrm{XPS}$ 谱图

Figure $4 \mathrm{Cu} 2 \mathrm{p} \mathrm{XPS}$ spectra of $x \mathrm{CuO} / \mathrm{CeO}_{2}-\mathrm{ZrO}_{2} / \mathrm{SiC}$ catalysts (a) $2 \% \mathrm{CuO} / \mathrm{CeO}_{2}-\mathrm{ZrO}_{2} / \mathrm{SiC}$; (b) $5 \% \mathrm{CuO} / \mathrm{CeO}_{2}-\mathrm{ZrO}_{2} / \mathrm{SiC}$; (c) $10 \% \mathrm{CuO} /$ $\mathrm{CeO}_{2}-\mathrm{ZrO}_{2} / \mathrm{SiC}$; (d) $20 \% \mathrm{CuO} / \mathrm{CeO}_{2}-\mathrm{ZrO}_{2} / \mathrm{SiC}$

图 5 为 $x \mathrm{CuO} / \mathrm{CeO}_{2}-\mathrm{ZrO}_{2} / \mathrm{SiC}$ 整体催化剂的 $\mathrm{Cu} \mathrm{LMM}$ 谱图. 如图所示, 915.4 916.7 eV 和 $911.8 \sim 914.2 \mathrm{eV}$ 两 处特征峰的结合能均低于 $918.6 \mathrm{eV}\left(\mathrm{Cu}^{0}\right.$ 的俄歇电子动 能), 因此两处特征峰均归属于 $\mathrm{Cu}^{+}$. 由此可见, 催化剂 表面存在 $\mathrm{Cu}^{2+}$ 和 $\mathrm{Cu}^{+}$两种 $\mathrm{Cu}$ 物种. 但 XRD 中并未出现
$\mathrm{Cu}_{2} \mathrm{O}$ 的特征衍射峰, 这是因为 $\mathrm{Cu}$ 物种总量较少, 且 $\mathrm{Cu}_{2} \mathrm{O}$ 大多以高度分散的形式存在于 $\mathrm{CeO}_{2}-\mathrm{ZrO}_{2}$ 固溶体 表面和孔道中. 另外, $\mathrm{Cu}^{+}$的出现是由于 $\mathrm{Cu}^{2+}$ 与 $\mathrm{CeO}_{2}-\mathrm{ZrO}_{2}$ 固溶体中的 $\mathrm{Ce}^{3^{+}}$发生相互作用造成的, 因此 $\mathrm{Cu}^{+}$的含量也体现了载体和活性组分之间的相互作用强 度. 如表 4 所示, $5 \% \mathrm{CuO} / \mathrm{CeO}_{2}-\mathrm{ZrO}_{2} / \mathrm{SiC}$ 催化剂中 $\mathrm{Cu}^{+}$ 含量较高, 载体与活性组分之间相互作用强度较强, 因 此催化活性较好. 而 $2 \% \mathrm{CuO} / \mathrm{CeO}_{2}-\mathrm{ZrO}_{2} / \mathrm{SiC}$ 催化剂中, 虽然 $\mathrm{Cu}^{+}$含量并不低, 但是因其 $\mathrm{Cu}$ 物种总负载量较少, 因此并没有展现出较好的催化活性.

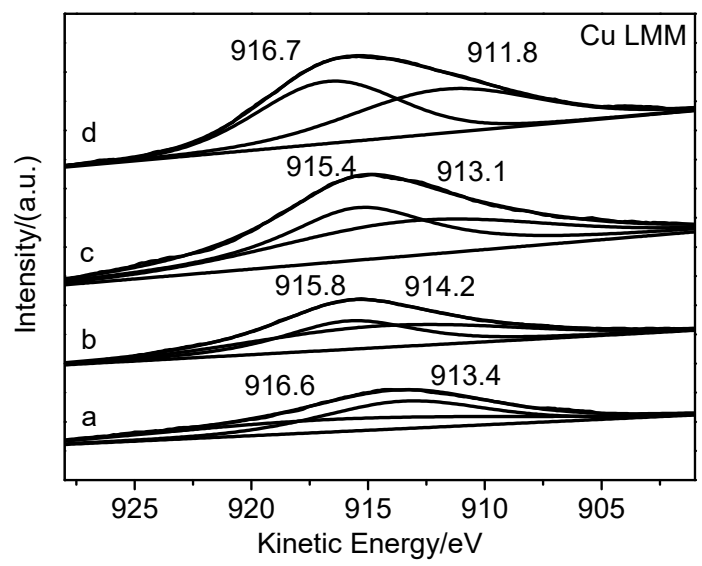

图 $5 x \mathrm{CuO} / \mathrm{CeO}_{2}-\mathrm{ZrO}_{2} / \mathrm{SiC}$ 整体催化剂 $\mathrm{Cu} \mathrm{LMM}$ 谱图 Figure $5 \mathrm{Cu} \mathrm{LMM}$ spectra of $x \mathrm{CuO} / \mathrm{CeO}_{2}-\mathrm{ZrO}_{2} / \mathrm{SiC}$ catalysts (a) $2 \% \mathrm{CuO} / \mathrm{CeO}_{2}-\mathrm{ZrO}_{2} / \mathrm{SiC}$; (b) $5 \% \mathrm{CuO} / \mathrm{CeO}_{2}-\mathrm{ZrO}_{2} / \mathrm{SiC}$; (c) $10 \% \mathrm{CuO}$ $\mathrm{CeO}_{2}-\mathrm{ZrO}_{2} / \mathrm{SiC}$; (d) $20 \% \mathrm{CuO} / \mathrm{CeO}_{2}-\mathrm{ZrO}_{2} / \mathrm{SiC}$

\subsubsection{Zr 3d XPS 分析}

图 6 为 $x \mathrm{CuO} / \mathrm{CeO}_{2}-\mathrm{ZrO}_{2} / \mathrm{SiC}$ 整体催化剂的 $\mathrm{Zr} 3 \mathrm{~d} \mathrm{X}$ 射线光电子能谱. 图中 $182 \mathrm{eV}$ 附近的特征峰和 $184 \mathrm{eV}$ 附近的特征峰分别对应 $\mathrm{Zr} 3 \mathrm{~d}_{5 / 2}$ 能级和 $\mathrm{Zr} 3 \mathrm{~d}_{3 / 2}$ 能级的电 子结合能, 均属于 $\mathrm{Zr}^{4+}$ 的特征峰. 说明催化剂中存在 $\mathrm{Zr}^{4}$ 物种.

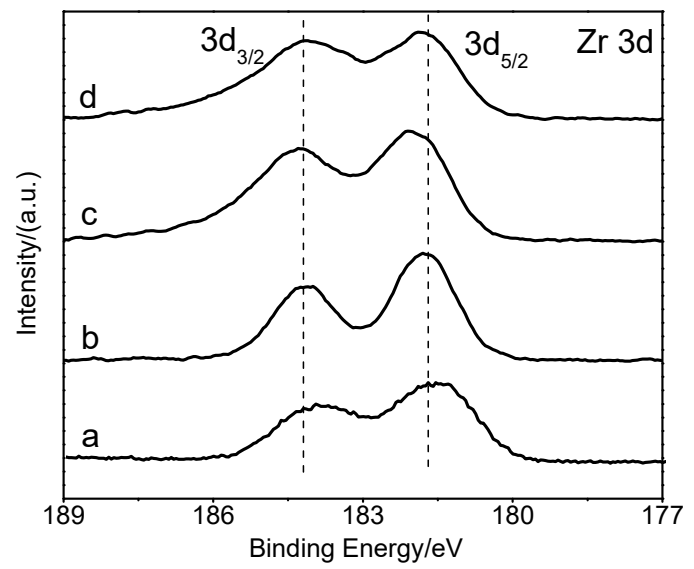

图 $6 x \mathrm{CuO} / \mathrm{CeO}_{2}-\mathrm{ZrO}_{2} / \mathrm{SiC}$ 整体催化剂 $\mathrm{Zr} 3 \mathrm{~d}$ XPS 谱图 Figure $6 \mathrm{Zr} 3 \mathrm{~d}$ XPS spectra of $x \mathrm{CuO} / \mathrm{CeO}_{2}-\mathrm{ZrO}_{2} / \mathrm{SiC}$ catalysts (a) $2 \% \mathrm{CuO} / \mathrm{CeO}_{2}-\mathrm{ZrO}_{2} / \mathrm{SiC}$; (b) $5 \% \mathrm{CuO} / \mathrm{CeO}_{2}-\mathrm{ZrO}_{2} / \mathrm{SiC}$; (c) $10 \% \mathrm{CuO}$ $\mathrm{CeO}_{2}-\mathrm{ZrO}_{2} / \mathrm{SiC}$; (d) $20 \% \mathrm{CuO} / \mathrm{CeO}_{2}-\mathrm{ZrO}_{2} / \mathrm{SiC}$ 


\subsubsection{O 1s XPS 分析}

图 7 为 $x \mathrm{CuO} / \mathrm{CeO}_{2}-\mathrm{ZrO}_{2} / \mathrm{SiC}$ 整体催化剂的 $\mathrm{O} 1 \mathrm{~s} \mathrm{X}$ 射线光电子能谱. 图中 $529.4 \sim 531.3 \mathrm{eV}$ 处的特征峰归 属于晶格氧的电子结合能, 而 $531.9 \sim 534.3 \mathrm{eV}$ 处的特 征峰则归属于吸附 $\mathrm{O}$ 物种的结合能. 除此之外, 随着 $\mathrm{Cu}$ 负载量的增加, 吸附氧的结合能逐渐增强, 这是由于 $\mathrm{Cu}$ 负载量的上升会使得催化剂中获得更多的能够吸附 $\mathrm{O}$ 物种的 $\mathrm{CuO}$, 因此使得吸附 $\mathrm{O}$ 物种的量增加.

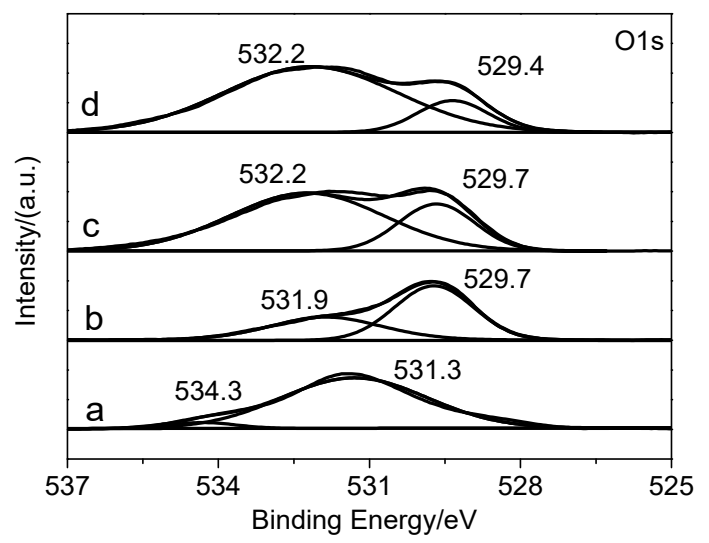

图 $7 x \mathrm{CuO} / \mathrm{CeO}_{2}-\mathrm{ZrO}_{2} / \mathrm{SiC}$ 整体催化剂 O 1s XPS 谱图

Figure $7 \mathrm{O}$ 1s XPS spectra of $x \mathrm{CuO} / \mathrm{CeO}_{2}-\mathrm{ZrO}_{2} / \mathrm{SiC}$ catalysts (a) $2 \% \mathrm{CuO} / \mathrm{CeO}_{2}-\mathrm{ZrO}_{2} / \mathrm{SiC}$; (b) $5 \% \mathrm{CuO} / \mathrm{CeO}_{2}-\mathrm{ZrO}_{2} / \mathrm{SiC}$; (c) $10 \% \mathrm{CuO} /$ $\mathrm{CeO}_{2}-\mathrm{ZrO}_{2} / \mathrm{SiC}$; (d) $20 \% \mathrm{CuO} / \mathrm{CeO}_{2}-\mathrm{ZrO}_{2} / \mathrm{SiC}$

\section{6 催化剂性能测试}

图 8 为 $x \mathrm{CuO} / \mathrm{CeO}_{2}-\mathrm{ZrO}_{2} / \mathrm{SiC}$ 整体催化剂在 $280 \sim$ $360{ }^{\circ} \mathrm{C}$ 温度范围内的甲醇水蒸气重整制氢催化活性图, 结果表明, $5 \% \mathrm{CuO} / \mathrm{CeO}_{2}-\mathrm{ZrO}_{2} / \mathrm{SiC}$ 催化剂催化活性较高, 在温度 $(T)$ 为 $360{ }^{\circ} \mathrm{C}$ 、水醇物质的量比 $\left(\mathrm{H}_{2} \mathrm{O} / \mathrm{CH}_{3} \mathrm{OH}\right)$ 为 1.2 : 1、甲醇水蒸气总空速(GHSV)为 $4840 \mathrm{~h}^{-1}$ 的条件下, 甲醇转化率为 $89.9 \%$. 其次为 $10 \% \mathrm{CuO} / \mathrm{CeO}_{2}-\mathrm{ZrO}_{2} / \mathrm{SiC}$ 催化剂, 甲醇转化率为 $83.8 \%$. 再次为 $20 \% \mathrm{CuO} /$ $\mathrm{CeO}_{2}-\mathrm{ZrO}_{2} / \mathrm{SiC}$ 催化剂, 甲醇转化率为 $80.7 \% .2 \% \mathrm{CuO} /$

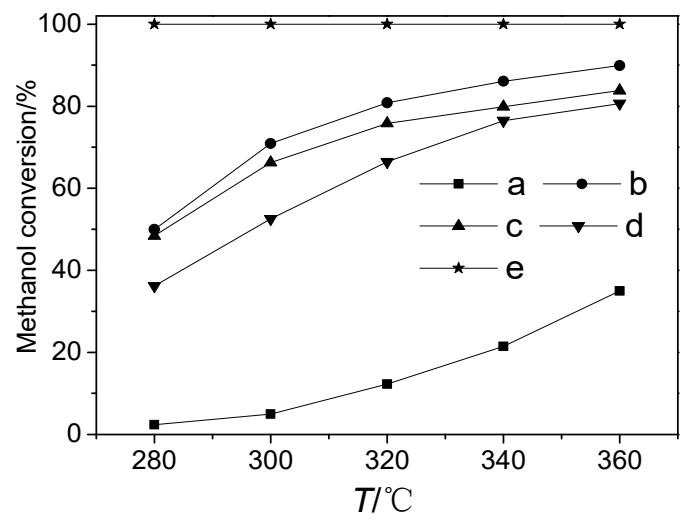

图 8 反应温度与催化剂性能的关系

Figure 8 Correlation between reaction temperature and catalyst performance

(a) $2 \% \mathrm{CuO} / \mathrm{CeO}_{2}-\mathrm{ZrO} \mathrm{Zr}_{2} / \mathrm{SiC}$; (b) $5 \% \mathrm{CuO} / \mathrm{CeO}_{2}-\mathrm{ZrO}_{2} / \mathrm{SiC}$; (c) $10 \% \mathrm{CuO} /$ $\mathrm{CeO}_{2}-\mathrm{ZrO}_{2} / \mathrm{SiC}$; (d) $20 \% \mathrm{CuO} / \mathrm{CeO}_{2}-\mathrm{ZrO}_{2} / \mathrm{SiC}$; (e) equilibrium. Reaction condition: $\mathrm{H}_{2} \mathrm{O} / \mathrm{CH}_{3} \mathrm{OH}=1.2$, GHSV $=4840 \mathrm{~h}^{-1}$
$\mathrm{CeO}_{2}-\mathrm{ZrO}_{2} / \mathrm{SiC}$ 催化剂催化活性最低，甲醇转化率为 $35.0 \%$. 结合表 $2 、$ 表 3 和表 4 可知, $5 \% \mathrm{CuO} / \mathrm{CeO}_{2}-\mathrm{ZrO}_{2} /$ $\mathrm{SiC}$ 催化剂 $\mathrm{Cu}$ 比表面积较大、 $\mathrm{CuO}$ 还原峰温度较低、 活性组分 $\mathrm{CuO}$ 与 $\mathrm{CeO}_{2}-\mathrm{ZrO}_{2}$ 固溶体之间相互作用较强、 氧空穴量较多, 因此催化活性较好. 而对于 $2 \% \mathrm{CuO} /$ $\mathrm{CeO}_{2}-\mathrm{ZrO}_{2} / \mathrm{SiC}$ 催化剂而言, 虽然 $\mathrm{Cu}$ 的分散较好, 活性 组分 $\mathrm{CuO}$ 与 $\mathrm{CeO}_{2}-\mathrm{ZrO}_{2}$ 固溶体之间相互作用较强, 但由 于 $\mathrm{CuO}$ 负载量较少, 导致 $\mathrm{Cu}$ 的比表面积较小、起到催 化活性的 $\mathrm{Cu}$ 物种总量较少, 并且氧空穴量较少, 因此 其催化活性较差. 综上所述, 催化剂 $\mathrm{Cu}$ 比表面积越大, $\mathrm{CuO}$ 还原温度越低, 活性组分 $\mathrm{CuO}$ 与 $\mathrm{CeO}_{2}-\mathrm{ZrO}_{2}$ 固溶体 之间相互作用越强, 氧空穴量越多, 催化剂的催化活性 越好.

提高 $\mathrm{H}_{2}$ 热效率的较好方法是将其通入质子交换膜 电池中, 但质子交换膜电池阳极的 $\mathrm{Pt}$ 电极对 $\mathrm{CO}$ 十分敏 感, 微量的 $\mathrm{CO}$ 就会使其出现中毒现象 ${ }^{[30-31]}$, 因此研究 重整尾气中 $\mathrm{CO}$ 摩尔含量具有重要意义. 图 9 给出了不 同反应温度下重整尾气中 $\mathrm{CO}$ 摩尔含量, 结果表明, $\mathrm{CO}$ 含量随着温度的升高而增加, 这是因为随着温度的升 高，甲醇更容易发生甲醇裂解反应，产生大量的 CO. 另 外, 温度上升使得水汽变换反应的化学平衡向逆向进 行, 进而抑制了 $\mathrm{CO}$ 转化为 $\mathrm{CO}_{2}$, 也可能对 $\mathrm{CO}$ 的增加有 一定贡献. 由图 9 可知, $10 \% \mathrm{CuO} / \mathrm{CeO}_{2}-\mathrm{ZrO}_{2} / \mathrm{SiC}$ 催化剂 重整尾气中 $\mathrm{CO}$ 摩尔含量较多, 为 $3.0 \%$. 其次为 $5 \% \mathrm{CuO} / \mathrm{CeO}_{2}-\mathrm{ZrO}_{2} / \mathrm{SiC}$ 催化剂, 重整尾气中 $\mathrm{CO}$ 摩尔含 量为 $2.8 \%$. 再次为 $20 \% \mathrm{CuO} / \mathrm{CeO}_{2}-\mathrm{ZrO}_{2} / \mathrm{SiC}$ 催化剂, 重 整尾气中 $\mathrm{CO}$ 摩尔含量为 $1.5 \% ; 2 \% \mathrm{CuO} / \mathrm{CeO}_{2}-\mathrm{ZrO}_{2} / \mathrm{SiC}$ 催化剂重整尾气中 $\mathrm{CO}$ 摩尔含量最少, 为 $1.0 \%$.

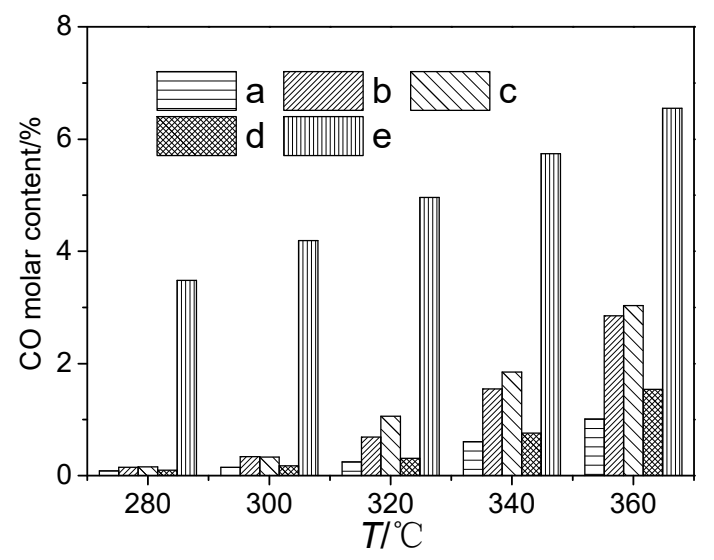

图 9 反应温度与重整尾气中 $\mathrm{CO}$ 摩尔含量的关系

Figure 9 Correlation between reaction temperature and CO molar content

(a) $2 \% \mathrm{CuO} / \mathrm{CeO}_{2}-\mathrm{ZrO}_{2} / \mathrm{SiC}$; (b) $5 \% \mathrm{CuO} / \mathrm{CeO}_{2}-\mathrm{ZrO}_{2} / \mathrm{SiC}$; (c) $10 \% \mathrm{CuO}$ $\mathrm{CeO}_{2}-\mathrm{ZrO}_{2} / \mathrm{SiC}$; (d) $20 \% \mathrm{CuO} / \mathrm{CeO}_{2}-\mathrm{ZrO}_{2} / \mathrm{SiC}$; (e) equilibrium. Reaction condition: $\mathrm{H}_{2} \mathrm{O} / \mathrm{CH}_{3} \mathrm{OH}=1.2$, $\mathrm{GHSV}=4840 \mathrm{~h}^{-1}$

表 5 为 $5 \% \mathrm{CuO} / \mathrm{CeO}_{2}-\mathrm{ZrO}_{2} / \mathrm{SiC}$ 整体催化剂与文献中 催化剂性能对比表。结果表明，本工作所制备的 $5 \% \mathrm{CuO} / \mathrm{CeO}_{2}-\mathrm{ZrO}_{2} / \mathrm{SiC}$ 整体催化剂在高空速下有较好 
的催化性能. 在甲醇水蒸气气体空速为 $4840 \mathrm{~h}^{-1}$, 反应 温度为 $360{ }^{\circ} \mathrm{C}$ 、水醇物质的量比为 $1.2: 1$ 的条件下, 产 氢速率高达 $1556 \mathrm{~L} \cdot \mathrm{m}^{-3} \cdot \mathrm{s}^{-1}$, 相较于前期研究的文献中 $\mathrm{CuZnCeZr}$ 颗粒催化剂 $1020 \mathrm{~L} \cdot \mathrm{m}^{-3} \cdot \mathrm{s}^{-1}$ 的产氢速率提升 了 $52.5 \%{ }^{[32]}$. 整体催化剂与颗粒催化剂相比, 具有反应 物扩散快、床层压力降小等优点, 因此在高空速下能表 现出良好的性能, 也解决了颗粒催化剂在工业生产中因 压力降大和温度场分布不均而造成的催化剂性能较差 的问题. 另外, 利用 $\mathrm{SiC}$ 整体催化材料可以直接制作成 反应器, 得益于 $\mathrm{SiC}$ 陶瓷材料优良的导电性和导热性该 反应器理论上可实现通电自热, 避免了传统颗粒催化剂 因外加反应器和加热带所带来的装置体积增大的问题, 为未来移动氢源系统的开发奠定了基础.

表 5 催化剂的产氢速率对比

Table 5 Hydrogen production rate of catalysts

\begin{tabular}{|c|c|c|c|c|}
\hline 催化剂 & $T /{ }^{\circ} \mathrm{C}$ & $\mathrm{H}_{2} \mathrm{O} / \mathrm{CH}_{3} \mathrm{OH}$ & $\begin{array}{c}\text { GHSV/ } \\
\mathrm{h}^{-1}\end{array}$ & $\begin{array}{l}\text { 产氢速率/ } \\
\left(\mathrm{L} \cdot \mathrm{m}^{-3} \cdot \mathrm{s}^{-1}\right)\end{array}$ \\
\hline $5 \% \mathrm{CuO} / \mathrm{CeO}_{2}-\mathrm{ZrO}_{2} / \mathrm{SiC}$ & 360 & $1.2: 1$ & 4840 & 1556 \\
\hline $10 \% \mathrm{Cu} / \gamma-\mathrm{Al} @ \mathrm{MMO}^{[24]}$ & 300 & $1.2: 1$ & 1760 & 721 \\
\hline $\mathrm{CuO} / \mathrm{CeO}_{2}-\mathrm{R}^{[26]}$ & 240 & $1.2: 1$ & 1760 & 631 \\
\hline $\mathrm{CuZnCeZr^{[32] }}$ & 240 & $1.2: 1$ & 2640 & 1020 \\
\hline $\mathrm{Zn}_{1} \mathrm{Ce}_{1} \mathrm{Zr}_{9} \mathrm{O}_{x}^{[33]}$ & 400 & $1.4: 1$ & 2955 & 1135 \\
\hline $\mathrm{Zn}_{5} \mathrm{Ce}_{1} \mathrm{Zr}_{9} \mathrm{O}_{x}^{[33]}$ & 400 & $1.4: 1$ & 2955 & 476 \\
\hline
\end{tabular}

\section{3 结论}

采用溶胶凝胶法制备了 $\mathrm{CeO}_{2}-\mathrm{ZrO}_{2}$ 固溶体, 并采用 浸渍法制备了性能良好且能适应大空速反应的 $x \mathrm{CuO} / \mathrm{CeO}_{2}-\mathrm{ZrO}_{2} / \mathrm{SiC}$ 整体催化剂。其中 $5 \% \mathrm{CuO} /$ $\mathrm{CeO}_{2}-\mathrm{ZrO}_{2} / \mathrm{SiC}$ 整体催化剂催化性能较好, 在反应温度 为 $360{ }^{\circ} \mathrm{C}$ 、水醇物质的量比为 1.2 、甲醇水蒸气气体空 速为 $4840 \mathrm{~h}^{-1}$ 的条件下, 甲醇转化率为 $89.9 \%$, 产氢速 率高达 $1556 \mathrm{~L} \cdot \mathrm{m}^{-3} \cdot \mathrm{s}^{-1}$. 另外催化剂活性的主要影响因 素为 $\mathrm{Cu}$ 比表面积、活性组分与载体之间相互作用强度 以及载体中的氧空穴量. 其中 $5 \% \mathrm{CuO} / \mathrm{CeO}_{2}-\mathrm{ZrO}_{2} / \mathrm{SiC}$ 整 体催化剂的 $\mathrm{Cu}$ 比表面积较大, 并且 $\mathrm{CuO}$ 与载体之间相 互作用较强, 因此表现出较好的催化性能. 除此之外, 整体催化剂相较于传统颗粒催化剂, 床层压力降小、反 应物扩散快, 因此具备良好的催化性能. 并且在 $\mathrm{SiC}$ 载 体材料良好的导电性导热性加持下, $\mathrm{CuO} / \mathrm{CeO}_{2}-\mathrm{ZrO}_{2} /$ $\mathrm{SiC}$ 整体催化剂能够在不外加加热装置的条件下集成为 反应器, 缩小了反应器的体积同时强化了其传热性能, 因此该整体催化剂具备极大的开发潜力.

\section{4 实验部分}

\section{1 催化剂制备}

\subsection{1 蜂窝 $\mathrm{SiC}$ 陶瓷的预处理}

将已经成型的蜂窝 $\mathrm{SiC}$ 陶瓷(贵州木易精细陶瓷有 限责任公司生产)浸泡在 5\%的 $\mathrm{HNO}_{3}$ 溶液中 $2 \mathrm{~h}$, 用去离 子水反复清洗干净, $120{ }^{\circ} \mathrm{C}$ 干燥 $2 \mathrm{~h}$, 在 $500{ }^{\circ} \mathrm{C}$ 马弗炉中
焙烧 $2 \mathrm{~h}$.

\subsection{2 $\mathrm{CeO}_{2}-\mathrm{ZrO}_{2} / \mathrm{SiC}$ 催化载体的制备}

配制 $1.6 \mathrm{~mol} / \mathrm{L} \quad \mathrm{Ce}\left(\mathrm{NO}_{3}\right)_{3} \cdot 6 \mathrm{H}_{2} \mathrm{O}$ 和 $0.4 \mathrm{~mol} / \mathrm{L}$ $\mathrm{Zr}\left(\mathrm{NO}_{3}\right)_{4} \cdot 5 \mathrm{H}_{2} \mathrm{O}$ 的混合溶液, 以 $180 \mathrm{r} / \mathrm{min}$ 的摚拌速度在 $30{ }^{\circ} \mathrm{C}$ 恒温水浴下滴加 $5 \%$ 的 $\mathrm{NH}_{3} \cdot \mathrm{H}_{2} \mathrm{O}$ 溶液, 形成凝胶 后, 以相同的搅拌速度滴加 $5 \%$ 的 $\mathrm{HNO}_{3}$ 溶液进行解胶, 直至凝胶变为溶胶时停止滴加. 把预处理过的蜂窝 $\mathrm{SiC}$ 陶瓷浸渍在溶胶中, $30 \mathrm{~min}$ 后取出, 吹去 $\mathrm{SiC}$ 孔内的残 液后, 放入 $120{ }^{\circ} \mathrm{C}$ 烘箱中干燥 $6 \mathrm{~h}, 500{ }^{\circ} \mathrm{C}$ 马弗炉中焙烧 $3 \mathrm{~h}$. 重复此操作, $\mathrm{CeO}_{2}-\mathrm{ZrO}_{2}$ 涂层涂覆次数为两次, 涂 层涂覆量(涂层质量占 $\mathrm{SiC}$ 的质量百分比)达到 $15 \% \pm$ $1 \%$.

\subsection{3 $\mathrm{CuO} / \mathrm{CeO}_{2}-\mathrm{ZrO}_{2} / \mathrm{SiC}$ 整体催化剂的制备}

将制备的 $\mathrm{CeO}_{2}-\mathrm{ZrO}_{2} / \mathrm{SiC}$ 催化剂载体材料浸渍在 $1.2 \mathrm{~mol} / \mathrm{L}$ 的 $\mathrm{Cu}\left(\mathrm{NO}_{3}\right)_{2} \cdot 3 \mathrm{H}_{2} \mathrm{O}$ 溶液中 $2 \mathrm{~h}$ 后取出, 在 $120{ }^{\circ} \mathrm{C}$ 烘箱中干燥 $6 \mathrm{~h}, 400{ }^{\circ} \mathrm{C}$ 马弗炉中焙烧 $3 \mathrm{~h}$. 重复 此操作, 直到 $\mathrm{CuO}$ 负载量 $(\mathrm{CuO}$ 质量含量占 $\mathrm{CeO}_{2}-\mathrm{ZrO}_{2} / \mathrm{SiC}$ 催化剂载体材料的质量百分比)为 $2 \% \pm$ $1 \% 、 5 \% \pm 1 \% 、 10 \% \pm 1 \% 、 20 \% \pm 1 \%$ ，分别命名为 $x \% \mathrm{CuO} / \mathrm{CeO}_{2}-\mathrm{ZrO}_{2} / \mathrm{SiC}$, 其中 $x=2,5,10$ 和 20 .

\section{2 催化剂性能评价}

催化剂性能评价在实验室自行搭建的固定床装置 上进行, 取 $1 \mathrm{~mL}$ 整体催化剂装入石英管固定床反应器 内，在 $280{ }^{\circ} \mathrm{C}$ 下用 $\mathrm{H}_{2}$ 体积分数为 $5 \% \mathrm{H}_{2}-\mathrm{N}_{2}$ 混合气体还 原 $2 \mathrm{~h}$, 切换为 $\mathrm{N}_{2}$ 吹扫并降至室温后关闭 $\mathrm{N}_{2}$, 调至反应 温度进行反应, 重整气经过冷凝器和干燥器脱除未反应 的甲醇和水后, 进入上海诜兴科学仪器有限公司的 1690 型气相色谱仪中, 用热导检测器(TCD)在线检测.

利用甲醇转化率和产氢速率评价催化剂性能. 具体 公式如下:

甲醇转化率 $(X)$ :

$$
\begin{aligned}
& X=\frac{F_{\mathrm{R}} \times\left(C_{\mathrm{CO}}+C_{\mathrm{CO}_{2}}\right) \times(32+18 w)}{(F \times \rho \times 22.4 \times 1000)} \times 100 \% \\
& \text { 产氢速率 }\left(Y_{\mathrm{H}_{2}}\right): \\
& Y_{\mathrm{H}_{2}}=\frac{F_{\mathrm{R}} \times C_{\mathrm{H}_{2}}}{60 \times V_{\text {cat }} \times 1000}
\end{aligned}
$$

$F_{\mathrm{R}}$ 为标况下重整尾气流量, $\mathrm{mL} \cdot \mathrm{min}^{-1} ; F$ 为泵设定的进 料量, $\mathrm{mL} \cdot \mathrm{min}^{-1} ; \rho$ 为甲醇和水混合溶液的密度, $\mathrm{g} \cdot \mathrm{mL}^{-1}$; $V_{\text {cat }}$ 为所用催化剂体积, $\mathrm{m}^{3} ; w$ 为水与甲醇物质的量比; $C_{\mathrm{CO}}$ 为重整尾气中 $\mathrm{CO}$ 的体积摩尔浓度, $\% ; C_{\mathrm{CO} 2}$ 为重整 尾气中 $\mathrm{CO}_{2}$ 的体积摩尔浓度, $\% ; C_{\mathrm{H} 2}$ 为重整尾气中 $\mathrm{H}_{2}$ 的体积摩尔浓度, \%.

\section{References}

[1] Jung, C.; Nagel, L.; Schindler, D.; Grau, L. Int. J. Hydrogen Energy 2018, 43, 23161.

[2] Muradov, N. Int. J. Hydrogen Energy 2017, 42, 14058.

[3] Uyar, T. S.; Besikci, D. Int. J. Hydrogen Energy 2017, 42, 2453. 
[4] Xi, H. J.; Hou, X. N.; Liu, Y. J.; Qing, S. J.; Gao, Z. X. Angew. Chem., Int. Ed. 2014, 53, 11886.

[5] Chi, H.; Andolina, C. M.; Li, J.; Curnan, M. T.; Saidi, W. A.; Zhou, G. W.; Yang, J. C.; Veser, G. Appl. Catal., A 2018, 556, 64.

[6] Jampa, S.; Jamieson, A. M.; Chaisuwan, T.; Luengnaruemithai, A.; Wongkasemjit, S. Int. J. Hydrogen Energy 2017, 42, 15073.

[7] Kuc, J.; Neumann, M.; Armbruster, M.; Yoon, S.; Zhang, Y. C.; Erni, R.; Weidenkaff, A.; Matam, S. K. Catal. Sci. Technol. 2016, 6, 43.

[8] Ma, Y. F.; Guan, G. Q.; Phanthong, P.; Li, X. M.; Cao, J.; Hao, X. G.; Wang, Z. D.; Abudula, A. Int. J. Hydrogen Energy 2014, 39, 18803.

[9] Sa, S.; Silva, H.; Brandao, L.; Sousa, J. M.; Mendes, A. Appl. Catal., $B$ 2010, 99, 43 .

[10] Yao, C. Z.; Zhang, X. R.; Wang, L. C.; Cao, Y.; Dai, W. L.; Fan, K. N.; Wu, D.; Sun, Y. H. Acta Chim. Sinica 2006, 64, 269 (in Chinese). (姚成漳, 张新荣, 王路存, 曹勇, 戴维林, 范康年, 吴东, 孙予 罕, 化学学报, 2006, 64, 269.)

[11] Kim, W.; Mohaideen, K. K.; Seo, D. J.; Yoon, W. L. Int. J. Hydrogen Energy 2017, 42, 2081.

[12] Zhou, J. J.; Zhang, Y.; Wu, G. S.; Mao, D. S.; Lu, G. Z. RSC $A d v$. 2016, 6,30176

[13] Das, D.; Llorca, J.; Dominguez, M.; Colussi, S.; Trovarelli, A.; Gayen, A. Int. J. Hydrogen Energy 2015, 40, 10463.

[14] Figueiredo, R. T.; Martinez-Arias, A.; Granados, M. L.; Fierro, J. L. G. J. Catal. 1998, 178, 146.

[15] Liu, Y.; Hayakawa, T.; Tsunoda, T.; Suzuki, K.; Hamakawa, S.; Murata, K.; Shiozaki, R.; Ishii, T.; Kumagai, M. Top. Catal. 2003, 22, 205.

[16] Khani, Y.; Bahadoran, F.; Safari, N.; Soltanali, S.; Taheri, S. A. Int. J. Hydrogen Energy 2019, 44, 11824.

[17] Zhou, S. L.; Yuan, Z. S.; Wang, S. D. Int. J. Hydrogen Energy 2006, $31,924$.

[18] Qi, A. D.; Wang, S. D.; Fu, G. Z.; Ni, C. J.; Wu, D. Y. Appl. Catal., A 2005, 281, 233.

[19] Suetsuna, T.; Suenaga, S.; Fukasawa, T. Appl. Catal., A 2004, 276, 275.

[20] Liu, N.; Yuan, Z. S.; Zhang, C. X.; Wang, S. J.; Li, D. Y.; Wang, S. D. Chin. J. Catal. 2005, 26, 1078 (in Chinese). (刘娜, 袁中山, 张 纯希, 王淑娟, 李德意, 王树东, 催化学报, 2005, 26, 1078.)
[21] Liu, N.; Wang, S. D.; Yuan, Z. S.; Zhang, C. X.; Wang, S. J.; Li, D. Y.; Fu, G. Z. CIESC J. 2004, 55, 90 (in Chinese). (刘娜, 王树东, 袁 中山, 张纯希, 王淑娟, 李德意, 付桂芝, 化工学报, 2004, 55, 90.)

[22] Liu, Y. J.; Wang, D. Z.; Zhang, L.; Bai, J.; Chen, L.; Liu, D. S. Fine Chem. 2018, 35, 2045 (in Chinese). (刘玉娟，王东哲，张否，白金， 陈琳，刘道胜，精细化工, 2018, 35, 2045.)

[23] Putna, E. S.; Bunluesin, T.; Fan, X. L.; Gorte, R. J.; Vohs, J. M.; Lakis, R. E.; Egami, T. Catal. Today 1999, 50, 343.

[24] He, J. P.; Yang, Z. X.; Zhang, L.; Li, Y.; Pan, L. W. Int. J. Hydrogen Energy 2017, 42, 9930.

[25] He, J. P.; Zhang, L.; Chen, L.; Yang, Z. X.; Tong, Y. F. Chem. J. Chinese U. 2017, 38, 1822 (in Chinese). (贺建平, 张磊, 陈琳, 杨 占旭, 佟宇飞, 高等学校化学学报, 2017, 38, 1822.)

[26] Yang, S. Q.; Zhou, F.; Liu, Y. J.; Zhang, L.; Chen, Y.; Wang, H. H.; Tian, Y.; Zhang, C. S.; Liu, D. S. Int. J. Hydrogen Energy 2019, 44, 7252 .

[27] She, W.; Qi, T. Q. J.; Cui, M. X.; Yan, P. F.; Weng, N. S. W.; Li, W. Z.; Li, G. M. ACS Appl. Mater. Interfaces 2018, 10, 14698.

[28] Wang, D. Z.; Feng, X.; Zhang, J.; Chen, L.; Zhang, L.; Wang, H. H.; Bai, J.; Zhang, C. S.; Zhang, Z. Y. J. Fuel Chem. Technol. 2019, 47 1251 (in Chinese). (王东哲, 冯旭, 张健, 陈琳, 张否, 王宏浩, 白 金, 张财顺, 张政一, 燃料化学学报, 2019, 47, 1251.)

[29] Prusty, D.; Pathak, A.; Mukherjee, M.; Mukherjee, B.; Chowdhury, A. Mater. Charact. 2015, 10, 31.

[30] Bai, Y. X.; Wu, J. J.; Qiu, X. P.; Wang, J. S.; Zhu, W. T.; Chen, L. Q. Acta Chim. Sinica 2006, 64, 527 (in Chinese). (白玉霞, 吴建军, 邱 新平, 王建设, 朱文涛, 陈立泉, 化学学报, 2006, 64, 527.)

[31] Wang, Z. H.; Shi, G. Y.; Xia, J. F.; Zhang, F. F.; Xia, Y. Z.; Li, Y. H.; Xia, L. H. Acta Chim. Sinica 2013, 71, 1225 (in Chinese). (王宗花, 史国玉，夏建飞，张菲菲，夏延致，李延辉，夏临华，化学学报, 2013, 71, 1225.)

[32] Zhang, L.; Pan, L. W.; Ni, C. J.; Sun, T. J.; Zhao, S. S.; Wang, S. D.; Wang, A. J.; Hu, Y. K. Int. J. Hydrogen Energy 2013, 38, 4397.

[33] Song, Q. L.; Men, Y.; Wang, J. G.; Liu, S.; Chai, S. S.; An, W.; Wang, K.; Li, Y. Y.; Tang, Y. H. Int. J. Hydrogen Energy 2020, 45 9592 .

(Cheng, B.) 